\title{
Focus on CRISPR tools and therapies
}

C

RISPR-based genome editing is now not only an established part of the standard repertoire of molecular biology, but also an important emerging therapeutic class entering clinical testing. CRISPR tools have proven indispensable for knock-in and knockout models, biological screens and understanding complex biology via multiplexed genetic screening. These methods are constantly being refined to further increase sensitivity and reproducibility and have already yielded invaluable insights into biology (see Review by Hanna and Doench).

Although Cas9 continues to be the most widely used endonuclease in research labs, its large size, reliance on double-strand breaks, off-target endonuclease activity and low efficiencies for gene replacement are propelling efforts to both discover new enzymes with favorable properties and engineer existing endonucleases with an increasingly diverse range of new activities. Some more recent examples include DNA base editing by dCas-deaminase fusion proteins, DNA integration by Cas $12 \mathrm{k}$ - or Cascade-transposase complexes, RNA base editing via the ADAR (adenosine deaminases acting on RNA) enzyme, and even transcriptional or epigenetic regulators linked to dCas (see Review by Anzalone et al.).

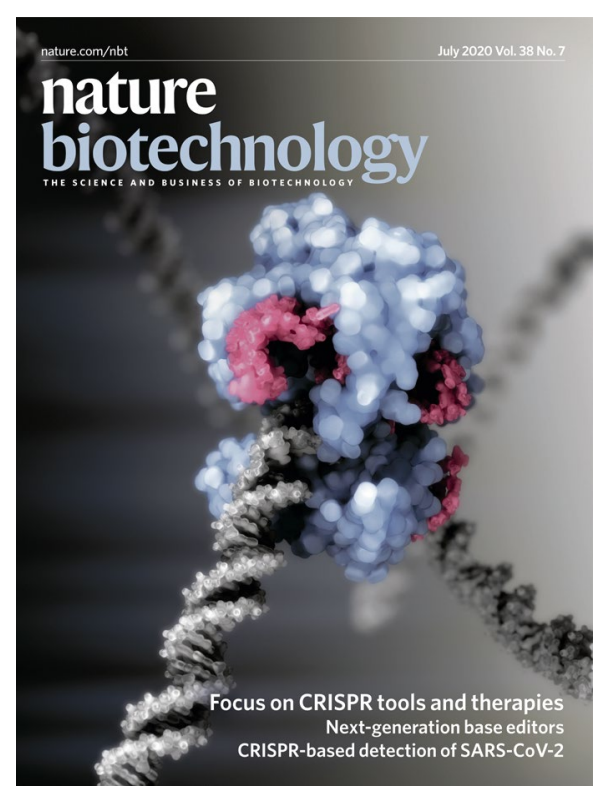

subretinally via an adeno-associated viral vector. Overall, for CRISPR therapies to be relevant in a much broader range of ailments, concerted efforts to optimize and innovate in delivery agents will be a necessity. In many respects, the key challenge will be to discover new means of delivering the CRISPR machinery to a broader range of target tissues with high efficiency (see Review by van Haasteren et al.)

A special episode of Nature Biotechnology's Forum podcast features Markus Elsner in conversation with Jennifer Doudna and David Liu about the promises and challenges of genome editing. In our First Rounders podcast, Brady Huggett interviews Katrine Bosley, former CEO of Editas Medicines and Avila Therapeutics. Bosley discusses growing up in Ohio, her first job in biotech (as an administrative assistant) and why her five years at CRISPR company Editas felt more like 1,000.

Finally, we present a snapshot of

As more of these bench tools move forward into translational programs, we can expect CRISPR agents to have a larger impact at the bedside (see Editorial). In a handful of clinical trials, somatic cells are being edited ex vivo and transfused into the patient bloodstream; one trial is also delivering Cas9 and its guide RNA recent patents related to CRISPR-based gene editing, disease treatment, and methods for targeting nucleic acids (see Patent Table).

Published online: 8 July 2020 https://doi.org/10.1038/s41587-020-0611-3 